
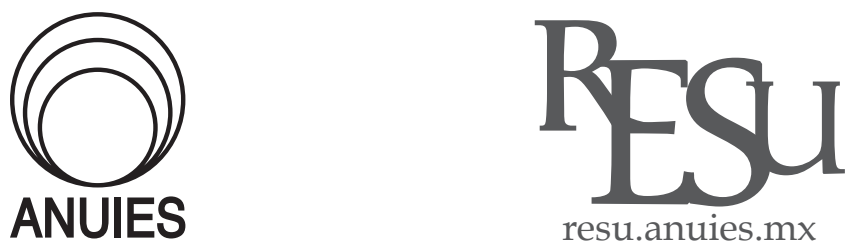

REVITA DEU
EDICACIÓN
SUPERIOR

ARTíCULO

\title{
Transformando la educación: análisis de diseños para la construcción de conocimiento por colaboración
}

Transforming higher education: designs analysis for collaborative construction of knowledge

\author{
Guillermo Santos* y Felipe Tirado** \\ * Universidad Nacional Autónoma de México-Facultad de Estudios Superiores Iztacala. \\ Correo electrónico: guillermosantos@comunidad.unam.mx \\ Recibido el 25 de septiembre del 2017; aceptado el 14 de febrero del 2019.
}

\section{Resumen}

En este trabajo se analizan procesos y resultados de un diseño educativo que utiliza mapas conceptuales, protocolos de colaboración y evaluación entre pares para la elaboración de un trabajo escrito. Se reportan diferencias en el desempeño en distintas métricas, analizadas a nivel individuo, equipo y grupo. A partir de los hallazgos se hacen sugerencias y se plantean pautas para mejorar el diseño educativo utilizado.

Palabras clave: Educación en Línea, Diseño Educativo, Aprendizaje por Colaboración, Protocolos de Colaboración, Co-Evaluación.

\begin{abstract}
In this paper we analyze the processes and results of an educational design that uses conceptual maps, collaboration protocols and peer evaluation for the preparation of a written work. Differences in performance are reported in different metrics, analyzed at the individual team and group level. From the findings, suggestions are made and guidelines are proposed to improve the educational design used.
\end{abstract}

Keywords: Online Education, Educational Design, Collaborative Learning, Scripts of Collaboration, Co-Evaluation. 


\section{Introducción}

—1 uso de las nuevas tecnologías de la información y la comunicación ha - 1 cobrado una importancia central para mejorar la calidad de vida de las personas en múltiples ámbitos. La educación representa un área en la que se aprecia la gran relevancia de su potencial para transformar la experiencia y la calidad de la educación de manera muy significativa.

La educación superior aún se encuentra fuertemente influenciada por modelos concebidos por la exposición del conocimiento, en vez de su reflexión y su utilización, lo que ha llevado a una educación memorística, por lo que en ocasiones se cuenta con pocas posibilidades de tener expresiones reflexivas, críticas y creativas por parte de los participantes.

Los sistemas de educación en línea y a distancia presentan importantes ventajas sobre los presenciales y abiertos (presencia esporádica), no obstante, existe evidencia de que su tasa de deserción y abandono suele ser mayor. El abandono suele ser atribuido a la falta de responsabilidad del estudiante, sin apreciarse que el problema es sistémico, en tanto también se debe a las características propias del entorno, de los profesores, de la cultura, de la concepción curricular y el diseño de los procesos educativos, al usar planteamientos que no facilitan el aprendizaje activo, la colaboración y la interacción discursiva entre pares y profesores (Meyer, 2014).

Por lo anterior, se vuelve fundamental generar conocimiento para la mejora de las prácticas de enseñanza-aprendizaje, identificando estrategias y diseños educativos que faciliten la formación de los estudiantes.

\section{Justificación}

La investigación educativa en entornos de aprendizaje mediados por cómputo ha tenido importantes avances en los últimos años, con la intención de poder comprender qué prácticas de uso son las que tienen mayor potencial, para mejorar los procesos de aprendizaje por colaboración (Stahl, Koschmann, \& Suthers, 2006).

Los primeros antecedentes en torno a ello demostraron que no era posible encontrar las condiciones ideales para que el aprendizaje por colaboración 
ocurriese (Dillenbourg, 1999). Los planteamientos evolucionaron hacia un enfoque probabilístico en donde, si bien se plantean una serie de expectativas de diseño para promover el aprendizaje, se reconoce que no es posible predecirlo. De aquí que se opte por intervenir directamente en el proceso, a manera de gestión, en tanto los entornos de aprendizaje por colaboración soportado por computadora son impredecibles, complejos y se encuentran poderosamente influidos por el contexto. Por ello se busca comprender de manera sistémica las relaciones entre condiciones, procesos y resultados (Dillenbourg, Järvelä, \& Fischer, 2009; P. A. Kirschner, 2002; P. Kirschner, Martens, \& Strijbos, 2004).

De acuerdo con Barab (2014), el enfoque de Investigación Basada en Diseño (Design-Based Research) se centra en la investigación y la innovación educativas, a partir de la idea de que tanto la teoría como la práctica emergen conjuntamente a través de diseños o intervenciones educativas. Éstas son puestas en marcha en una lógica de iteración, de tal manera que se pueda reconocer cuándo, cómo, y por qué funcionan determinados diseños, además de resaltar los elementos que permitan mejorarlos. En esa lógica, se torna importante comprender los procesos de aprendizaje y aquellas situaciones contextuales que afectan el comportamiento de nuestro diseño. De esta manera, además de desarrollar teoría, se pueden usar los resultados para obtener una serie de pautas que permitan mejorar las implementaciones que se realizan.

Por lo anterior, cobra relevancia para este estudio contar con evidencia que permita entender el funcionamiento del diseño educativo empleado, para poder realizar mejoras en sus ediciones futuras, además de valorar su impacto en los procesos y resultados de aprendizaje.

\section{Objetivo}

El objetivo del presente trabajo es analizar un diseño educativo que fue aplicado en dos cursos universitarios, en términos de procesos y resultados, tanto a nivel de grupo como de equipo e individuo. La finalidad es encontrar aquellos elementos que permitan mejorar el diseño, al identificar las características del grupo, de los equipos y de los alumnos que obtienen el mejor desempeño en las que tareas que se prescribieron. También se explora la relación entre distintos indicadores de desempeño, con un instrumento de evaluación del proceso de colaboración. 


\section{Pregunta de investigación}

¿Qué tanto influye el diseño educativo en el proceso y sus resultados de aprendizaje, al incorporar distintos niveles de análisis (grupo - equipo - individuo)?

\section{Hipótesis}

- Existen tendencias de desempeño en el cuestionario del proceso de la colaboración, de acuerdo al grupo, el equipo y el alumno.

- Existen tendencias de desempeño en la participación en los foros para la construcción del ensayo de acuerdo al grupo, el equipo y el alumno.

- Existen tendencias de desempeño en torno a la calificación obtenida en los ensayos de acuerdo al grupo, el equipo y el alumno.

- Existen tendencias de desempeño en torno al tiempo que los alumnos invierten en la plataforma de acuerdo al grupo, el equipo y el alumno.

- Existe una relación entre el desempeño en la elaboración de productos de aprendizaje de equipo y alumno, con la evaluación del proceso de la colaboración.

- Existe una relación entre el desempeño en la elaboración de productos de aprendizaje de equipo y alumno, con el tiempo de actividad en el aula virtual.

\section{Marco teórico}

Las Tecnologías de la Información y la Comunicación (TIC) son medios con enormes potencialidades, por lo que hay que reconocer y crear condiciones para que éstas sean incorporadas a las prácticas educativas (Kirschner, 2002). A este respecto, conviene entender a las TIC como vehículos fundamentales de la experiencia educativa, siempre y cuando seamos capaces de reconocer las mejores prácticas de uso de las mismas, lo cual se vuelve un paso fundamental para avanzar hacia una concepción sobre la mejora de la educación mediada por tecnología que permita aprovechar sus grandes potencialidades a través de la intercomunicación (Coll et al., 2008).

\section{Diseño educativo y mejores prácticas de uso de la tecnología para la educación}

El desarrollo de experiencias educativas con potencial para mejorar el aprendizaje, se encuentra estrechamente relacionado con lo que llamamos diseño educati- 
vo. De acuerdo con Dennen y Hoadley (2013) podemos entender diseño educativo como la identificación y puesta en marcha de técnicas, estrategias y procedimientos, que nos permitan facilitar ciertos tipos de aprendizajes. De este modo se puede orientar la práctica educativa a través de pautas que permitan utilizar distintos medios, situaciones y criterios de implementación, que aprovechen dimensiones cognitivas, afectivas, sociales y culturales de los estudiantes, en beneficio de la enseñanza y el aprendizaje (Reigeluth, 2000).

Autores como Collins (1991), Pea (1997), y Greeno (2006; 1998), plantean que ciertas características de la práctica educativa son capaces de influir sobre el aprendizaje de las personas. Estas características deben ser diseñadas, por lo que señalan como punto de partida la creación de escenarios que involucren estratégicamente a los agentes que integran la actividad educativa (personas, recursos materiales e intelectuales), descentralizando la función educativa, que en el pasado se adjudicaba exclusivamente al profesor.

La tecnología requiere saber crear entornos de aprendizaje eficaces, que permitan que los alumnos aprendan los unos de los otros (enseñanza distribuida), aprovechando el conocimiento y la inteligencia compartida (cognición distribuida) (Cole y Engeström, 1993). Se deben crear estrategias de integración de equipos de colaboración, plantear objetivos, diseñar tareas desestructuradas (sin rutas definidas de solución) y precisar protocolos (guías) que orienten la interacción discursiva (argumentación / contra-argumentación), de manera que los alumnos interactúen con sus pares al construir conocimiento y resolver problemas significativos de su entorno. Una manera de cristalizar la construcción de conocimiento, es por medio de mapas conceptuales y ensayos elaborados a través de una dinámica de equipos de trabajo.

Los mapas conceptuales son una meta-herramienta desarrollada para favorecer el aprendizaje a partir de la irradiación conceptual que forma modelos de representación de conocimiento complejos, utilizando la articulación semántica conceptual por medio de conectores que confieren una sintaxis organizada y representada de forma gráfica (Novak y Cañas, 2006).

Otra estrategia ampliamente utilizada en la educación para que los alumnos desarrollen y expresen el conocimiento, es la elaboración de ensayos. En un ensayo se desarrolla un texto de manera libre, respetando una estructura básica que consiste en una introducción, el desarrollo de un planteamiento a partir de argumentos y elementos que les den sustento, finalizando con una conclusión. En el ensayo se puede exponer, analizar, interpretar y evaluar un planteamiento en torno a un tema central. Por ello el ensayo ofrece una estructura apropiada para la construcción de conocimiento basada en la argumentación sustentada, como se propone en las tesis de Toulmin (2003) antes referidas. 
Los ensayos pueden ser desarrollados en equipo, incorporando tareas que permitan aplicar el conocimiento a situaciones o problemas auténticos. Podemos considerar dicha tarea como una estrategia de aprendizaje por colaboración, ya que de acuerdo con la literatura de investigación (Strijbos, Martens y Jochems, 2004), la mayor parte de los entornos de aprendizaje orientados a la colaboración se basan en tareas abiertas, desestructuradas, sin solución única, y con amplio potencial para generar interacción.

Múltiples autores que han investigado el fenómeno del aprendizaje por colaboración, han hecho importantes aportaciones al diseño educativo. Se ha encontrado que determinadas formas de interacción discursiva, a partir de cierto tipo de preguntas, elaboración de explicaciones para otros, plantear hipótesis, construir argumentos, contra-argumentos y refutar, influyen de manera significativa en los resultados y procesos del aprendizaje, por ejemplo en la solución de conflictos socio-cognitivos, el aprendizaje de contenidos a nivel de dominio, la mejora de procesos de organización de tareas de grupo, entre otros aspectos (Fawcett \& Garton, 2005; Mugny \& Doise, 2006; O’Donnell \& King, 1999; Palincsar \& Brown, 1986; Roschelle \& Teasley, 1995; Sharan, 1980; Tudge, 1992; Webb, 1989).

Si bien la investigación ha identificado que ciertas formas de interactuar muestran potentes resultados al favorecer el aprendizaje, otras señalan que ciertas interacciones no lo hacen, porque en la manera natural y espontánea de la interacción, el nivel de discusión que se desarrolla suele ser muy superficial para llegar rápidamente al consenso (Cohen, 1994), en tanto les resulta lo más fácil. Määttä y colaboradores (2012) también señalan que los alumnos suelen llevar a cabo interacciones irrelevantes y fuera de contexto, cuando intentan resolver tareas de enseñanza-aprendizaje.

Se ha encontrado también que la colaboración arbitraria (o no sistematizada), puede derivar en niveles muy primarios de construcción de conocimiento que no trascienden la comparación de información (Gunawardena, 1997). De acuerdo con Laat (2002), esto se observa cuando se examinan el discurso en comunidades virtuales de aprendizaje.

\section{Protocolos de colaboración}

Se requiere formular propuestas en materia de diseño educativo partiendo de la idea de que es posible estructurar directamente los procesos interactivos de los estudiantes cuando colaboran. Es posible articular tales procesos utilizando lo que se ha denominado protocolos de colaboración (collaboration scripts).

De acuerdo a la literatura especializada, un protocolo de colaboración o guión colaborativo puede definirse como un artefacto de mediación que estructura 
y estimula los procesos interactivos entre dos o más compañeros de aprendizaje, al especificar objetivos, roles, planos sociales, secuencias de actividades, plazos temporales, formas de interacción, uso de recursos y metas o productos terminales (Dillenbourg \& Jermann, 2007; Dillenbourg, 2002; Kobbe et al., 2007; Kollar, Fischer, \& Hesse, 2006).

Existe evidencia de que el uso de guiones tiene efectos importantes sobre el aprendizaje de los alumnos, favoreciendo la comprensión del material de estudio, la solución de problemas, la adquisición de habilidades de argumentación, el conocimiento de dominio, la solución de conflictos socio-cognitivos y el entendimiento mutuo, entre otros. (Ploetzner, Dillenbourg, Preier, \& Traum, 1999; Weinberger, Stegmann, \& Fischer, 2007). Es posible estructurar patrones discursivos en la interacción social, para promover la actividad epistémica en la colaboración, ya que se encuentran relacionados con ciertas pautas que les indican a los estudiantes cómo trabajar con el conocimiento a nivel conceptual, por ejemplo, hacer preguntas, contra-argumentar o reelaborar los planteamientos del otro (Weinberger, Ertl, Fischer, \& Mandl, 2005).

En este trabajo, sostenemos que es posible reconocer la actividad epistémica o de construcción de conocimiento, como los intercambios discursivos que los alumnos manifiestan en torno a los contenidos curriculares. Es posible operacionalizar dicha actividad, registrando las acciones que los alumnos llevan a cabo cuando utilizan los artefactos de comunicación, en las discusiones que tienen al elaborar los productos de aprendizaje.

\section{Influencia Educativa Distribuida}

Existe una noción llamada influencia educativa distribuida (IED), la cual establece categorías para el análisis de la interactividad en línea, poniendo como foco de interés la influencia que alumnos y profesores ejercen entre sí (¿Quién debe hacer qué? ¿Para qué? ¿Sobre qué? ¿Con qué recursos?), con el fin de construir conocimiento en torno a los temas curriculares (Coll, Onrubia, \& Majós, 2008; Colomina, Onrubia, \& Rochera, 2007). Esta noción centra su análisis en los mecanismos de ayuda mutua que son contextuales a las necesidades de los aprendices y refuerzan su actividad constructiva.

Existen trabajos en el contexto de la educación en línea y a distancia que muestran que cuando se trata de foros de discusión es posible reconocer las acciones de influencia educativa más relevantes, para el apoyo a la actividad constructiva entre estudiantes (Coll, Bustos, \& Engel, 2015; Coll et al., 2015; Coll, Onrubia, et al., 2008).

La influencia educativa distribuida se plantea con base en tres categorías analíticas que se manifiestan en los intercambios comunicativos y son: 
- Gestión de la participación social: Reglas que determinan la actuación social de los participantes.

- Gestión de la tarea: Indicaciones sobre la forma en la que se realizará la tarea, características, procedimientos y productos.

- Construcción de significados: Proceso de construcción de conocimiento compartido entre los estudiantes, con una relación directa a los contenidos de enseñanza-aprendizaje.

En este trabajo se hace un esfuerzo por emplear estas categorías, no sólo como un recurso de análisis, sino como un medio para el diseño de los protocolos de colaboración. También se emplean en la construcción de un cuestionario para reconocer si las distintas categorías están relacionadas con el desempeño de los alumnos, tanto en la actividad individual como por colaboración.

\section{Planteamiento del Problema}

Las reflexiones antes desarrolladas destacan la necesidad de que la educación mediada por tecnología cuente con diseños educativos adecuados. Se requiere generar compromiso, responsabilidad y procesos discursivos reflexivos en los estudiantes, para resolver las tareas de enseñanza-aprendizaje en formas productiva (Scardamalia, 2002). Para ello se requiere la implementación de un diseño educativo que apoye los procesos de colaboración regulando la interacción social (por ejemplo, la organización, la calidad de la tarea o construcción de conocimiento). Se busca que fluya la capacidad creativa y propositiva de los alumnos, de tal forma que den lugar a una dinámica de enseñanza y aprendizaje distribuidos, lo cual es posible ofreciendo información a todos los participantes de manera que puedan reconocer e interpretar el comportamiento colaborativo (Dillenbourg, 2005; Jermann, Soller, \& Muehlenbrock, 2005).

Considerando las razones anteriores se vuelve fundamental contar con un marco que permita el diseño de experiencias para enriquecer el aprendizaje con dinámicas basadas en la colaboración. Una estrategia que se ha mostrado útil para involucrar a los alumnos en dinámicas de enseñanza-aprendizaje por colaboración es la co-evaluación o evaluación por pares, en la cual un equipo de alumnos evalúa el trabajo de otro equipo y viceversa, emitiendo argumentos sobre lo evaluado. Para tener una base con referentes comunes, se emplean rúbricas de evaluación en las que se especifican los criterios a evaluar y los niveles de desempeño en tales criterios. 


\section{Método}

Este estudio se basa en el trabajo realizado con dos grupos de estudiantes pertenecientes al primer semestre de la carrera Psicología en el Sistema de Universidad Abierta y Educación a Distancia (SUAyED) de la UNAM, correspondiente al semestre escolar 2017-1.

Se trabajó en dos módulos, uno, el de Sistemas Teóricos de la Psicología, y el otro, en el de Introducción a la Filosofía de la Psicología. En el primer módulo participaron 21 estudiantes y en el segundo (Introducción a la Filosofía de la Psicología) se inscribieron 38 estudiantes. Estos módulos forman parte de los fundamentos disciplinarios de la psicología, por lo que se ubican en el plan de estudios en la primera etapa denominada plataforma única de conocimientos básicos, de la carrera SUAyED psicología. Para efectos del análisis de datos, denominaremos al grupo de Sistemas Teóricos de la Psicología con las siglas STP, y al grupo Introducción a la Filosofía de la Psicología con las siglas IFP.

Se planteó un estudio de caso en cada uno de los módulos referidos (Sistemas Teóricos de la Psicología - Introducción a la Filosofía de la Psicología), a partir de un enfoque de investigación basada en diseño. A partir del trabajo en línea, se incorporaron los estudiantes de ambos módulos a un diseño educativo que tuvo tres componentes: 1) La elaboración de mapas conceptuales por cada unidad temática de los contenidos curriculares del curso. 2) El seguimiento de protocolos de colaboración para guiar las dinámicas de interacción discursiva, a fin de construir un ensayo por colaboración en equipo. 3) La co-evaluación tanto de los mapas conceptuales como producto individual, así como del ensayo desarrollado por colaboración en equipo.

\section{Procedimiento}

A continuación, se describe el procedimiento de selección de equipos de trabajo:

- En ambos grupos se conformaron equipos de trabajo (3 en STP y 4 en IFP), con 4 a 5 integrantes para la elaboración de un ensayo por colaboración, como producto en el que se expresa la construcción de conocimiento.

- Los equipos fueron nominados por letras (A, B y C en stP y A, B, C y D en IFP).

- A efecto de integrar equipos de trabajo con un cierto equilibrio y promover que fueran heterogéneos en su calidad académica, se tomaron en cuenta diversas métricas de desempeño, tales como calificación obtenida en el bachillerato, tiempo en plataforma, acceso a los recursos y calificación en 
actividad de encuadre, de modo que cada equipo fuera integrado por un alumno de desempeño alto, otro medio y un bajo, donde unos ayudan a los otros.

\section{Encuadre del curso}

- Cada equipo consultó los recursos de encuadre (syllabus) que incluyó el calendario escolar, un video de bienvenida, un calendario de actividades del curso, documentos sobre dinámica de trabajo y de la evaluación, así como un documento que explica el sentido y la estrategia de trabajo. También se entregó, en la plataforma digital, un artículo sobre la fundamentación y el proceso de elaboración de mapas conceptuales y el acceso a la herramienta digital Cmap Tools, con la que éstos se desarrollan.

El curso constó de cinco unidades, las cuales se desarrollaron durante 15 semanas. Cada una de las unidades tomó tres semanas para realizar las actividades correspondientes. Cada unidad fue desarrollada incorporando los elementos de diseño educativo de la siguiente forma (ver tabla 3):

- La primera semana se destinó a la entrega y la co-evaluación de un mapa conceptual a partir de un material bibliográfico relacionado con la temática de dicha unidad.

- En el caso de STP, durante la segunda y la tercer semanas se destinaron a la elaboración por equipo de un micro-ensayo, en el cual se describiera el objeto de estudio, los principales planteamientos conceptuales, así como sus alcances y limitaciones. En el caso del curso IFP, el ensayo trató sobre el desarrollo de un cuestionamiento relacionado con algún aspecto central de epistemología de la ciencia, así como el planteamiento de ejemplos y argumentos relacionados con tal aspecto. El objetivo de la tarea es diferente en cada uno de los cursos.

- Al terminar cada ensayo, éste fue co-evaluado por alumnos de otro equipo, evitando que se hicieran evaluaciones recíprocas, es decir, que no se evaluaran unos a otros (ej. equipo A evalúa a B, B a C, y C a A). Para ello se veló la identidad (de evaluados y evaluadores), de manera que no se dieran complicidades (ayudarse en la calificación) o rivalidades (castigar la calificación).

- Al finalizar el curso se aplicó el cuestionario de evaluación del proceso de la colaboración.

- Se desarrolló un protocolo de colaboración para llevar a cabo las discusiones en los foros relacionadas con el desarrollo del trabajo en equipo (ver tabla 1). También se dio otro protocolo para sugerir las acciones relacionadas con la construcción del trabajo por colaboración un Wiki (ver tabla 2). 
Tabla 1

Protocolo de colaboración para la interacción en foros de discusión

\begin{tabular}{lll}
\hline Valor & Etiqueta & Descripción \\
\hline 1 & Estoy de acuerdo & $\begin{array}{l}\text { Tu contribución únicamente muestra acuerdo a la } \\
\text { opinión de uno o varios de tus compañeros, sin } \\
\text { más argumentos. }\end{array}$ \\
\hline 2 & Doy mi punto de vista & $\begin{array}{l}\text { Tu contribución muestra tu propio punto de vista } \\
\text { respecto a la contribución de uno o varios de tus } \\
\text { compañeros. }\end{array}$ \\
\hline 3 & Doy mi punto de vista informado & $\begin{array}{l}\text { Tu contribución muestra argumentos críticos a uno } \\
\text { o varios planteamientos de tus compañeros, ha- } \\
\text { ciendo referencia a conocimiento de la(s) lectura(s) } \\
\text { revisada(s). }\end{array}$ \\
\hline 4 & Doy mi punto de vista crítico, informado & $\begin{array}{l}\text { Tu contribución muestra una opinión propia, } \\
\text { bien elaborada, y crítica tomando en cuenta una } \\
\text { y propositivo }\end{array}$ \\
& $\begin{array}{l}\text { la(s) lectura(s) revisada(s), además de proponer } \\
\text { acciones de mejora. }\end{array}$ \\
\hline
\end{tabular}

Tabla 2

Protocolo de colaboración para la construcción del ensayo

\begin{tabular}{|c|c|c|}
\hline $\begin{array}{c}\text { Ayudar en la } \\
\text { organización del trabajo }\end{array}$ & $\begin{array}{c}\text { Ayudar en la mejora } \\
\text { de la calidad de las tareas }\end{array}$ & $\begin{array}{c}\text { Ayudar en la construcción } \\
\text { colaborativa de conocimiento }\end{array}$ \\
\hline $\begin{array}{l}\text { - Realizan propuestas de } \\
\text { organización del trabajo en equipo. } \\
\text { - Participación en las discusiones } \\
\text { para la toma de decisiones de } \\
\text { organización en las herramientas } \\
\text { de comunicación. } \\
\text { - Participan en la coordinación de } \\
\text { la elaboración de los productos } \\
\text { escritos (qué, quiénes, cuándo y con } \\
\text { qué frecuencia se hará). } \\
\text { - Revisan plazos, avances y } \\
\text { monitorean si se trabaja de acuerdo } \\
\text { lo previsto. } \\
\text { - Atienden asuntos y conflictos de } \\
\text { organización oportunamente. }\end{array}$ & $\begin{array}{l}\text { - Revisan la evolución } \\
\text { del documento para } \\
\text { detectar consistencias e } \\
\text { inconsistencias, según las } \\
\text { características de lo que se } \\
\text { les pidió. } \\
\text { - Hacen comentarios } \\
\text { y sugerencias a los } \\
\text { compañeros, para la } \\
\text { mejora del trabajo después } \\
\text { de su revisión (cómo } \\
\text { hacerlo, qué características } \\
\text { de los productos debe } \\
\text { tener el trabajo de equipo). } \\
\text { - Responden preguntas } \\
\text { relacionadas a las } \\
\text { características de los } \\
\text { productos que debe tener } \\
\text { el trabajo de equipo. } \\
\text { - Plantean preguntas } \\
\text { relacionadas a las } \\
\text { características de la tarea, } \\
\text { su abordaje, producto y/o } \\
\text { resultados. }\end{array}$ & $\begin{array}{l}\text { - Revisan el historial de versiones } \\
\text { y cuestionan las bases teóricas y } \\
\text { empíricas de las aportaciones de } \\
\text { los compañeros en el documento } \\
\text { (usando el Foro de Discusión). } \\
\text { - Proporcionan una opinión propia } \\
\text { sobre los aspectos teóricos o } \\
\text { prácticos del trabajo. } \\
\text { - Proporcionan una opinión apoyada } \\
\text { en recursos bibliográficos (libros, } \\
\text { capítulos de libros, artículos, } \\
\text { investigaciones, etc.). } \\
\text { - Toman en cuenta las opiniones de } \\
\text { los compañeros para expresar su } \\
\text { propio punto de vista. } \\
\text { - Toman en cuenta las opiniones de } \\
\text { sus compañeros, para apoyarlas o } \\
\text { cuestionarlas basándose en fuentes } \\
\text { bibliográficas. } \\
\text { - Plantean a sus compañeros puntos } \\
\text { de discusión o reflexión, para } \\
\text { movilizar la interacción y mejorar } \\
\text { en equipo la estructura y contenido } \\
\text { del ensayo. }\end{array}$ \\
\hline
\end{tabular}

Tabla 3

Elementos de diseño educativo que se incluyeron en cada experiencia

Experiencia 2017-1

\begin{tabular}{|c|c|c|}
\hline Curso o Módulo Analizado & $\begin{array}{l}\text { Sistemas Teóricos de la Psicología } \\
\text { (sTP) }\end{array}$ & $\begin{array}{l}\text { Intriducción a la Filosofía de la } \\
\text { Psicología (IFP) }\end{array}$ \\
\hline Tipo de tarea & $\begin{array}{l}\text { Micro-ensayo sobre teorías } \\
\text { psicológicas }\end{array}$ & $\begin{array}{l}\text { Micro-ensayos sobre epistemología } \\
\text { de la ciencia }\end{array}$ \\
\hline
\end{tabular}

Continúa... 


\begin{tabular}{|c|c|c|}
\hline \multicolumn{3}{|c|}{ Experiencia 2017-1 } \\
\hline $\begin{array}{l}\text { Distribución de actividades } \\
\text { de elaboración colaborativa de } \\
\text { documento }\end{array}$ & $\begin{array}{l}\text { Planteada en varias experiencias dist } \\
\text { (micro-ensayos) }\end{array}$ & buidas a lo largo de todo el semestre \\
\hline Método de evaluación de producto & $\begin{array}{l}\text { Rúbrica para evaluar el análisis } \\
\text { teórico y planteamiento de ejemplos } \\
\text { de cada teoría y argumentación de } \\
\text { alcances y limitaciones }\end{array}$ & $\begin{array}{l}\text { Rúbrica para evaluar la respuesta } \\
\text { a un aspecto relevante sobre } \\
\text { epistemología de la ciencia, y un } \\
\text { ejemplo argumentado en torno a tal } \\
\text { aspecto }\end{array}$ \\
\hline $\begin{array}{l}\text { Evaluación de frecuencia de } \\
\text { participación }\end{array}$ & \multicolumn{2}{|c|}{ Sí } \\
\hline $\begin{array}{l}\text { Evalluación de contenido de } \\
\text { participación (protocolo) }\end{array}$ & \multicolumn{2}{|c|}{ Sí } \\
\hline Reglas de participación en los foros & \multicolumn{2}{|c|}{$\begin{array}{l}\text { Expectativa mínima de } 4 \text { intervenciones por semana en las actividades de } \\
\text { construcción colaborativa de ensayo }\end{array}$} \\
\hline Objeto de discusión & \multicolumn{2}{|c|}{$\begin{array}{l}\text { Construcción de un micro-ensayo por colaboración en equipo en cada } \\
\text { unidad, en torno a los aspectos preescritos para la elaboración de tal ensayo. }\end{array}$} \\
\hline
\end{tabular}

\section{Indicadores a analizar}

Se utilizaron las siguientes métricas de referencia para poder llevar a cabo cada uno de los análisis:

- Resultados de desempeño en el cuestionario de evaluación del proceso de la colaboración.

- Resultados de la participación en los foros de discusión.

- Calificación obtenida en mapas conceptuales.

- Calificación obtenida en ensayos por colaboración.

- Tiempo en plataforma (acciones de navegación en línea, en intervalos no mayores a 15 minutos).

\section{Resultados}

Los análisis se describen en tres niveles: curso, equipo e individuo.

\section{Nivel Curso}

\section{Proceso de Colaboración (nivel curso)}

Se obtuvo el porcentaje de desempeño de acuerdo al promedio de calificaciones recibidas por los evaluadores, en cada una de las dimensiones del cuestionario de evaluación del proceso de la colaboración. En la comparación entre ambos cursos a este respecto, podemos reconocer que el de "Introducción a la Filosofía de la Psicología" fue el que mejor desempeño obtuvo en las tres dimensiones del cuestionario del proceso de colaboración, alcanzando 
su mayor puntaje en la categoría de "Organización del trabajo" (OT), seguido de la de "Contribución en la Elaboración del Ensayo" (EE) y finalmente "Construcción de Conocimiento" (CC). Esto indica que los alumnos se involucraron mejor en acciones relacionadas con organizar el trabajo de equipo. En el caso del curso "La Psicología Científica y sus Sistemas Teóricos", podemos apreciar un menor desempeño general en las tres categorías del cuestionario, observándose el mayor puntaje en "OT", seguido de "EE" y "CC". Es de resaltar también que la mayor variabilidad en el caso del curso STP, se encontró en la dimensión de "EE", lo cual indica que en esta dimensión los alumnos tuvieron las intervenciones menos equitativas según su propio juicio, teniendo participaciones más equitativas en el indicador "OT". En el caso del curso IFP, es interesante observar que se aprecia por la desviación estándar que hay una mayor dispersión en la evaluación de EE (ver tabla 4).

Tabla 4

Comparación entre el porcentaje obtenido en las tres dimensiones y el total en el cuestionario de proceso de la colaboración en ambos cursos

\begin{tabular}{|c|c|c|c|c|c|}
\hline \multicolumn{2}{|c|}{$\begin{array}{l}\text { Curso al que pertenecen los } \\
\text { alumnos }\end{array}$} & $\begin{array}{c}\text { Porcentaje } \\
\text { Organización } \\
\text { del Trabajo (от) }\end{array}$ & $\begin{array}{c}\text { Porcentaje } \\
\text { Contribución } \\
\text { en la } \\
\text { Elaboración del } \\
\text { Ensayo (EE) }\end{array}$ & $\begin{array}{c}\text { Porcentaje } \\
\text { Construcción } \\
\text { de } \\
\text { Conocimiento } \\
\text { (cc) }\end{array}$ & $\begin{array}{c}\text { Porcentaje de } \\
\text { la Evaluación } \\
\text { del Proceso de } \\
\text { la Colaboración } \\
\text { (тот) }\end{array}$ \\
\hline \multirow{3}{*}{$\begin{array}{l}\text { 0104-La Psicología } \\
\text { Científica y sus } \\
\text { Sistemas Teóricos } \\
\text { (sTP) }\end{array}$} & Media & 79.51 & 77.16 & 72.99 & 75.96 \\
\hline & $\mathrm{N}$ & 9 & 9 & 9 & 9 \\
\hline & $\begin{array}{l}\text { Desviación } \\
\text { estándar }\end{array}$ & 11.41 & 17.76 & 16.42 & 14.95 \\
\hline \multirow{3}{*}{$\begin{array}{l}\text { 0101-Introducción } \\
\text { a la Filosofía de la } \\
\text { Psicología (IFP) }\end{array}$} & Media & 84.78 & 83.12 & 83.87 & 83.97 \\
\hline & $\mathrm{N}$ & 13 & 13 & 13 & 13 \\
\hline & $\begin{array}{l}\text { Desviación } \\
\text { estándar }\end{array}$ & 10.43 & 11.58 & 10.33 & 10.44 \\
\hline \multirow[t]{3}{*}{ Total } & Media & 82.62 & 80.68 & 79.42 & 80.70 \\
\hline & $\mathrm{N}$ & 22 & 22 & 22 & 22 \\
\hline & $\begin{array}{l}\text { Desviación } \\
\text { estándar }\end{array}$ & 10.90 & 14.34 & 13.92 & 12.80 \\
\hline
\end{tabular}

\section{Participación en Foros de Discusión (nivel curso)}

Se obtuvo la frecuencia de participación en los foros dedicados a cada actividad de discusión en el curso, y posteriormente el promedio por curso. En la comparación en torno a este aspecto, podemos encontrar que no se observó una tendencia uniforme en torno a la equidad de la participación a lo largo de los foros. En el caso de STP el primer foro tuvo un promedio de frecuencia de participación muy modesto (3.58 intervenciones en promedio), mientras que en el segundo foro aumentó significativamente $(8.07=125.4 \%)$, disminu- 
yendo en el tercero, aumentando en el cuarto, y volviendo a disminuir en el quinto. En el caso de IFP, la tendencia fue de menor a mayor en los primeros dos foros, para observarse una baja progresiva en el tercero, cuarto y quinto foros de discusión. Cabe mencionar que el primer foro no estuvo asociado a la elaboración de un ensayo, sino a la respuesta a un cuestionamiento planteado para generar polémica en torno a la unidad introductoria revisada en ambos cursos, y duró solo una semana, mientras que la duración de la participación en los foros de la unidad 2, 3, 4, y 5, fue de dos semanas, lo que permite explicar el aumento antes referido. En el caso de la participación en los foros de ensayo, se pidió a los alumnos como requerimiento participar al menos 4 veces por semana (un mínimo de 8 veces por ensayo), lo que indica que la mayor parte de los alumnos intentaron acercarse al estándar, puesto que los valores de la desviación estándar no presentaron grandes diferencias en los foros relacionados con el ensayo (ver tabla 5).

Tabla 5

Comparación entre el porcentaje obtenido en la participación en foros de discusión de cada uno de los equipos en ambos cursos

\begin{tabular}{|c|c|c|c|c|c|c|}
\hline \multicolumn{2}{|c|}{$\begin{array}{l}\text { Curso al que pertenecen los } \\
\text { alumnos }\end{array}$} & $\begin{array}{c}\text { Foro } \\
\text { Unidad 1 - } \\
\text { Frecuencia }\end{array}$ & $\begin{array}{c}\text { Foro } \\
\text { Unidad } 2 \text { - } \\
\text { Frecuencia }\end{array}$ & $\begin{array}{c}\text { Foro } \\
\text { Unidad } 3 \text { - } \\
\text { Frecuencia }\end{array}$ & $\begin{array}{c}\text { Foro } \\
\text { Unidad } 4 \text { - } \\
\text { Frecuencia }\end{array}$ & $\begin{array}{c}\text { Foro } \\
\text { Unidad } 5 \text { - } \\
\text { Frecuencia }\end{array}$ \\
\hline \multirow{3}{*}{$\begin{array}{l}\text { 0104-La Psicología } \\
\text { Científica y sus } \\
\text { Sistemas Teóricos } \\
\text { (sTP) }\end{array}$} & Media & 3.58 & 8.07 & 6.50 & 8.60 & 6.00 \\
\hline & $\mathrm{N}$ & 12 & 14 & 12 & 10 & 10 \\
\hline & $\begin{array}{l}\text { Desviación } \\
\text { estándar }\end{array}$ & 1.93 & 4.03 & 4.62 & 4.35 & 4.29 \\
\hline \multirow{3}{*}{$\begin{array}{l}\text { 0101-Introducción } \\
\text { a la Filosofía de la } \\
\text { Psicología (IFP) }\end{array}$} & Media & 2.83 & 9.07 & 8.21 & 4.89 & 5.08 \\
\hline & $\mathrm{N}$ & 18 & 14 & 14 & 9 & 12 \\
\hline & $\begin{array}{l}\text { Desviación } \\
\text { estándar }\end{array}$ & 1.54 & 4.67 & 4.41 & 2.57 & 3.15 \\
\hline \multirow[t]{3}{*}{ Total } & Media & 3.13 & 8.57 & 7.42 & 6.84 & 5.50 \\
\hline & $\mathrm{N}$ & 30 & 28 & 26 & 19 & 22 \\
\hline & $\begin{array}{l}\text { Desviación } \\
\text { estándar }\end{array}$ & 1.72 & 4.31 & 4.50 & 4.00 & 3.65 \\
\hline
\end{tabular}

Por lo mismo, es interesante apreciar el efecto del diseño educativo, ya que los alumnos tienden a generar ritmos de trabajo en los términos que se les indican, en este caso, cumplir con al menos 4 participaciones por semana. Esto es relevante porque rompe los patrones típicos observados, donde los estudiantes levantan su tasa de participación sólo cuando se acerca el fin del curso escolar. 


\section{Calificación en los Ensayos (nivel curso)}

La calificación en el ensayo se calculó promediando las emitidas por cada evaluador al ensayo de equipo asignado. En la mayor parte de los casos las calificaciones se construyeron por al menos 3 evaluaciones. En el análisis entre cursos, es importante destacar que el curso que menores calificaciones obtuvo en todos sus ensayos fue el IFP, disminuyendo las calificaciones de manera descendente en cada uno de sus ensayos. En lo respectivo al curso STP la calificación en el primer ensayo es relativamente alta (87.1), en el segundo caso disminuye (78.9) en el tercer caso, aumenta ligeramente (79.5) y en el último caso desciende, siendo la más baja (77.4) (ver tabla 6).

Tabla 6

Comparación entre el porcentaje de calificación en cada uno de los ensayos en ambos cursos

\begin{tabular}{|c|c|c|c|c|c|}
\hline \multicolumn{2}{|c|}{$\begin{array}{l}\text { Curso al que pertenecen los } \\
\text { alumnos }\end{array}$} & $\begin{array}{c}\text { Resultados } \\
\text { Coevaluación } \\
\text { Ensayo } \\
\text { Unidad } 2 \\
\end{array}$ & $\begin{array}{c}\text { Resultados } \\
\text { Coevaluación } \\
\text { Ensayo } \\
\text { Unidad } 3 \\
\end{array}$ & $\begin{array}{c}\text { Resultados } \\
\text { Coevaluación } \\
\text { Ensayo } \\
\text { Unidad } 4\end{array}$ & \multirow{2}{*}{$\begin{array}{c}\text { Resultados } \\
\text { Coevaluación } \\
\text { Ensayo } \\
\text { Unidad } 5 \\
77.40\end{array}$} \\
\hline $\begin{array}{l}\text { 0104-La Psicología } \\
\text { Científica y sus }\end{array}$ & Media & 87.08 & 78.91 & 79.50 & \\
\hline Sistemas Teóricos & $\mathrm{N}$ & 12 & 11 & 10 & 10 \\
\hline & $\begin{array}{l}\text { Desviación } \\
\text { estándar }\end{array}$ & 5.518 & 7.778 & 14.842 & 15.167 \\
\hline \multirow{3}{*}{$\begin{array}{l}\text { 0101-Introducción } \\
\text { a la Filosofía de la } \\
\text { Psicología (IFP) }\end{array}$} & Media & 86.08 & 77.54 & 74.62 & 67.54 \\
\hline & $\mathrm{N}$ & 13 & 13 & 13 & 13 \\
\hline & $\begin{array}{l}\text { Desviación } \\
\text { estándar }\end{array}$ & 4.310 & 27.153 & 18.081 & 22.919 \\
\hline \multirow[t]{3}{*}{ Total } & Media & 86.56 & 78.17 & 76.74 & 71.83 \\
\hline & $\mathrm{N}$ & 25 & 24 & 23 & 23 \\
\hline & $\begin{array}{l}\text { Desviación } \\
\text { estándar }\end{array}$ & 4.848 & 20.284 & 16.570 & 20.140 \\
\hline
\end{tabular}

De lo anterior podemos concluir que los cursos muestran un efecto de disminución progresiva en la calificación, conforme avanzan los ejercicios de ensayo. Esto se puede explicar porque los alumnos se esmeran menos en los últimos ejercicios de ensayo, que en los primeros. Pero hay otro componente, los contenidos en IFP son de mayores complejidad y extensión, lo que podría explicar que sea también mayor el deterioro observado en las calificaciones.

\section{Tiempo en Plataforma (nivel curso)}

Se calculó el tiempo que los alumnos inscritos en cada curso dedicaron a la realización de sus actividades (en intervalos mayores a 15 minutos entre clics, no contabiliza), para poder obtener el promedio por curso. En el caso de la comparación en torno al tiempo que dedicaron en promedio, tenemos una 
diferencia muy sutil entre ambos (1.37 horas) a favor del curso STP. En cuanto a la desviación estándar, tenemos que el curso IFP tuvo mayor variabilidad en torno al tiempo que dedicaron los alumnos. Cabe destacar que sólo se consideraron los casos que aprobaron el curso (es decir participaron en la mayor parte de la experiencia) y dedicaron más de 10 horas (ver tabla 7).

Tabla 7

Comparación entre el promedio de dedicación en horas en cada curso

\begin{tabular}{lccc}
$\begin{array}{l}\text { Curso al que pertenecen los } \\
\text { alumnos }\end{array}$ & Media & N & $\begin{array}{c}\text { Desviación } \\
\text { estándar }\end{array}$ \\
\hline $\begin{array}{l}\text { 0104-La Psicología Científica y sus } \\
\text { Sistemas Teóricos (STP) }\end{array}$ & 22.5823 & 13 & 8.50341 \\
\hline $\begin{array}{l}\text { 0101-Introducción a la Filosofía de la } \\
\text { Psicología (IFP) }\end{array}$ & 21.2118 & 17 & 10.45448 \\
\hline Total & 21.8057 & 30 & 9.52360 \\
\hline
\end{tabular}

Es interesante apreciar que a pesar de que los contenidos en IFP son más complejos y extensos, los alumnos invirtieron cantidades de tiempo similares, aunque como ya se señaló, obtuvieron calificaciones más bajas.

\section{Nivel Equipo}

\section{Proceso de Colaboración (nivel equipo-Curso STP)}

Se promediaron las calificaciones obtenidas de la coevaluación en cada uno de los ítems del cuestionario del proceso de colaboración que hicieron referencia a un compañero en particular (cada compañero tuvo entre 2 y 3 calificaciones que evaluaron su desempeño).

Posteriormente se realizó la sumatoria y se calculó el porcentaje obtenido en cada dimensión examinada a través del instrumento, así como el porcentaje total, considerando como el 100\% la calificación máxima que fue posible obtener en cada dimensión y en la totalidad de los reactivos del cuestionario.

Examinando el desempeño de los equipos del curso STP, se encontró que la dimensión de "Organización del trabajo en equipo" (от) fue en la que mejor se desempeñaron los equipos, seguida por "Contribución en la elaboración del ensayo" (EE), y por último, "Construcción de conocimiento" (CC). Esto indica que las acciones de los compañeros en el proceso de elaboración del trabajo de equipo guardan mayor relación con actividades de coordinación $\mathrm{y}$ acuerdos en el equipo en torno a las actividades (establecimiento de fechas, compromisos, delegación de responsabilidades), que con actividades 
de monitoreo del avance del documento y construcción del conocimiento en el mismo. Por último, es importante mencionar que el equipo $C$ fue el que mejor desempeño obtuvo en todos los indicadores siguiendo la tendencia antes mencionada (ver tabla 8).

Tabla 8

Muestra el promedio obtenido por cada equipo en cada dimensión y en total en el cuestionario del proceso de la colaboración en el curso STP

\begin{tabular}{llcccc}
\hline $\begin{array}{l}\text { Equipo al que } \\
\text { pertenecen los } \\
\text { alumnos }\end{array}$ & $\begin{array}{c}\text { Porcentaje } \\
\text { Organización del } \\
\text { Trabajo }\end{array}$ & $\begin{array}{c}\text { Porcentaje } \\
\text { Contribución en } \\
\text { la Elaboración del } \\
\text { Ensayo }\end{array}$ & $\begin{array}{c}\text { Porcentaje } \\
\text { Construcción de } \\
\text { Conocimiento }\end{array}$ & $\begin{array}{c}\text { Porcentaje de la } \\
\text { Evaluación del } \\
\text { Proceso de la } \\
\text { Colaboración }\end{array}$ \\
\hline A & Media & 69.5 & 64.6 & 59.4 & 63.7 \\
\cline { 2 - 6 } N & 4 & 4 & 4 & 4 \\
\hline Media & 93.8 & 100.0 & 1 & 1 \\
\cline { 2 - 6 } & $\begin{array}{l}\text { N (no válida } \\
\text { para el } \\
\text { análisis) }\end{array}$ & 1 & 1 & 80.9 & 83.2 \\
\hline D & Media & 85.9 & 84.0 & 4 & 4 \\
\cline { 2 - 6 } & N & 4 & 4 & & 4 \\
\hline
\end{tabular}

Se requiere indicar que en el caso de STP, los integrantes del equipo B abandonaron el curso.

Se aprecia en los valores de la tabla que el equipo $C$ fue el que mejor se integró en las tareas de colaboración, a diferencia del equipo A. Probablemente una problemática en la distribución del trabajo en equipo sea la heterogeneidad de los mismos, pudiendo haber una distribución no equilibrada, donde alumnos de bajo o alto rendimiento queden concentrados en un equipo. Aunque la distribución se hizo considerando alumnos de alto, medio y bajo rendimiento, probablemente habría que hacer una recomposición en la medida en la que se va observando el comportamiento de los integrantes del equipo.

De lo anterior, se invita a considerar que el cuestionario de evaluación del proceso de la colaboración podría utilizarse como instrumento de diagnóstico desde etapas tempranas, aplicándose más de una vez, conforme los equipos resuelven las tareas de enseñanza-aprendizaje, de manera que se cuente con indicadores que permitan recomponer los equipos de trabajo.

De acuerdo al enfoque de Investigación Basada en Diseño, precisamente recomienda el que se hagan ajustes con base en los resultados observados y no esperar a que una situación que no está operando de manera apropiada continúe. 


\section{Proceso de Colaboración (nivel equipo-Curso IFP)}

Examinando el desempeño de los equipos del curso IFP, se encontró que la dimensión de "Organización del trabajo en equipo" fue en la que mejor desempeño obtuvieron los equipos. No obstante, es importante destacar que los equipos A y C fueron los que mejor desempeño tuvieron en todas las dimensiones representadas en la tabla (ver tabla 9).

Tabla 9

Muestra el promedio obtenido por cada equipo en cada dimensión y en total en el cuestionario del proceso de la colaboración en el curso IFP

\begin{tabular}{|c|c|c|c|c|c|}
\hline \multicolumn{2}{|c|}{$\begin{array}{l}\text { Equipo al que } \\
\text { pertenecen los } \\
\text { alumnos }\end{array}$} & \multirow{2}{*}{$\begin{array}{c}\text { Porcentaje } \\
\begin{array}{c}\text { Organización del } \\
\text { Trabajo }\end{array} \\
88.5\end{array}$} & \multirow{2}{*}{$\begin{array}{c}\text { Porcentaje } \\
\text { Contribución en } \\
\text { la Elaboración del } \\
\text { Ensayo } \\
86.1\end{array}$} & \multirow{2}{*}{$\begin{array}{c}\text { Porcentaje } \\
\text { Construcción de } \\
\text { Conocimiento }\end{array}$} & \multirow{2}{*}{$\begin{array}{c}\text { Porcentaje de la } \\
\text { Evaluación del } \\
\text { Proceso de la } \\
\text { Colaboración } \\
87.5\end{array}$} \\
\hline A & Media & & & & \\
\hline & $\mathrm{N}$ & 3 & 3 & 3 & 3 \\
\hline \multirow[t]{2}{*}{ B } & Media & 78.9 & 77.1 & 79.7 & 78.8 \\
\hline & $\mathrm{N}$ & 4 & 4 & 4 & 4 \\
\hline \multirow[t]{2}{*}{ C } & Media & 91.1 & 88.9 & 88.2 & 89.3 \\
\hline & $\mathrm{N}$ & 4 & 4 & 4 & 4 \\
\hline \multirow[t]{2}{*}{ D } & Media & 78.1 & 79.2 & 78.1 & 78.4 \\
\hline & $\mathrm{N}$ & 2 & 2 & 2 & 2 \\
\hline
\end{tabular}

Nuevamente se aprecia en el caso del curso IFP que hay una heterogeneidad en el desempeño de los equipos, aunque no tan marcada con el caso de STP, pero igualmente teniendo presentes los planteamientos de la Investigación Basada en Diseño, podría ser conveniente la recomposición de los equipos.

\section{Participación en Foros de Discusión (nivel equipo-Curso STP)}

Se calcularon los promedios de la frecuencia de participación en cada equipo en cada uno de los foros de discusión, encontrando que en el curso sTP no existe un patrón consistente en el desempeño en cada uno de los foros, pues algunos tienen calificaciones altas y otros bajas, sin embargo vemos que en el foro de ensayo de la unidad 2, los equipos A, C y D presentan un desempeño alto, el cual decae posteriormente obteniendo uno medio, lo que no sería de esperar si se considera que los estudiantes hacia el final del curso suelen intensificar su trabajo (ver tabla 10 y gráfica 1). 
Tabla 10

Muestra el promedio obtenido por cada equipo en torno a la frecuencia de participación en cada foro de discusión en el curso STP

\begin{tabular}{lcccccc}
\hline $\begin{array}{l}\text { Equipo al que pertenecen } \\
\text { los alumnos }\end{array}$ & $\begin{array}{c}\text { Foro } \\
\text { Unidad 1 - } \\
\text { Frecuencia }\end{array}$ & $\begin{array}{c}\text { Foro } \\
\text { Unidad 2 } \\
\text { Frecuencia }\end{array}$ & $\begin{array}{c}\text { Foro } \\
\text { Unidad 3 - } \\
\text { Frecuencia }\end{array}$ & $\begin{array}{c}\text { Foro } \\
\text { Unidad 4 - } \\
\text { Frecuencia }\end{array}$ & $\begin{array}{c}\text { Foro } \\
\text { Unidad 5 - } \\
\text { Frecuencia }\end{array}$ \\
\hline A & Media & 4.5 & 10. & 5.8 & 6.0 & 6.0 \\
\cline { 2 - 7 } & $\mathrm{N}$ & 4 & 4 & 4 & 3.0 & 3.5 \\
\hline $\mathrm{C}$ & Media & 2.7 & 6.0 & 3 & 2 & 2 \\
\cline { 2 - 7 } & $\mathrm{N}$ & 3 & 4 & 9.6 & 11.6 & 7.0 \\
\hline $\mathrm{D}$ & $\mathrm{N}$ & 4.0 & 9.6 & 5 & 5 & 5 \\
\hline
\end{tabular}

\section{Gráfica 1}

Promedio obtenido por cada equipo en torno a la frecuencia de participación en cada foro de discusión en el curso STP

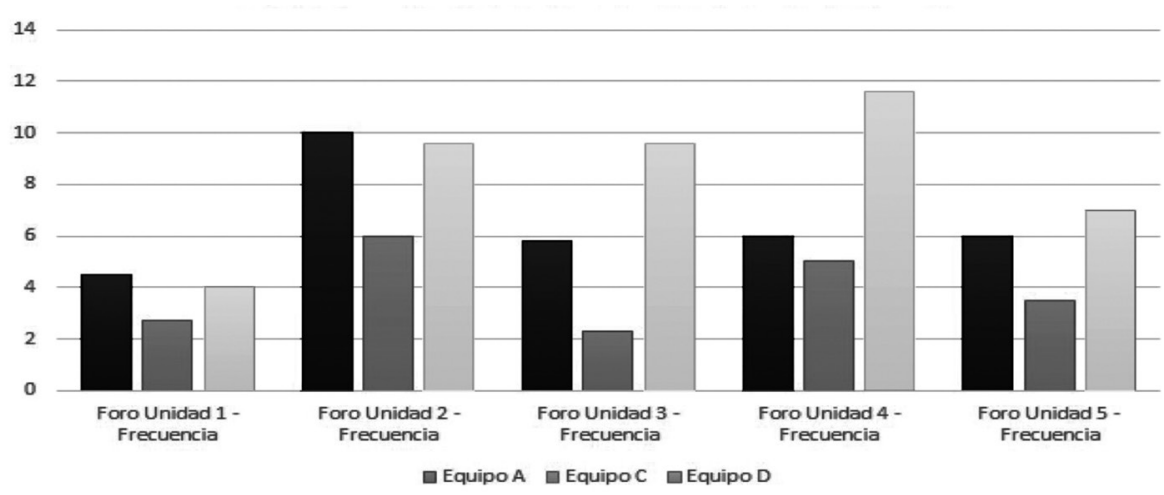

En la tabla 10 se puede apreciar claramente esta heterogeneidad del trabajo de los equipos, observando que hay un equipo que siempre fue el de más bajo desempeño (equipo C), el cual contrasta con el equipo D que obtuvo alto desempeño en la mayor parte de los foros. Cabe puntualizar que en los foros se genera la argumentación y la contra argumentación para la elaboración del ensayo, de ahí que reflejen indicadores de la actividad epistémica del equipo.

\section{Participación en Foros de Discusión (nivel equipo-Curso IFP)}

En el caso de los foros del curso IFP, es importante mencionar que el primer foro tiene un desempeño de participación bajo, sin embargo, en los primeros tres equipos los foros de la unidad 2 y 3 tienen un desempeño alto (excepto 
en el equipo B), el cual decae los foros de la unidad 4 y 5 respectivamente (ver tabla 11 y gráfica 2).

Tabla 11

Muestra el promedio obtenido por cada equipo en torno a la frecuencia de participación en cada foro de discusión en el curso IFP

\begin{tabular}{llccccc}
\hline $\begin{array}{l}\text { Equipo al que pertenecen } \\
\text { los alumnos }\end{array}$ & $\begin{array}{c}\text { Foro } \\
\text { Unidad 1 - } \\
\text { Frecuencia }\end{array}$ & $\begin{array}{c}\text { Foro } \\
\text { Unidad 2 - } \\
\text { Frecuencia }\end{array}$ & $\begin{array}{c}\text { Foro } \\
\text { Unidad 3 - } \\
\text { Frecuencia }\end{array}$ & $\begin{array}{c}\text { Foro } \\
\text { Unidad 4 - } \\
\text { Frecuencia }\end{array}$ & $\begin{array}{c}\text { Foro } \\
\text { Unidad 5 - } \\
\text { Frecuencia }\end{array}$ \\
\hline A & Media & 2.3 & 8.8 & 10.7 & 3.0 & 5.3 \\
\cline { 2 - 7 } & $\mathrm{N}$ & 3 & 4 & 3 & 2 & 3 \\
\hline B & Media & 4.3 & 9.0 & 4.3 & 6.3 & 6.3 \\
\cline { 2 - 7 } & $\mathrm{N}$ & 3 & 4 & 3 & 2 & 2 \\
\hline C & Media & 2.8 & 14.0 & 10.0 & 4.8 & 4.0 \\
\hline N & 4 & 4 & 4 & & 4 \\
\hline
\end{tabular}

Gráfica 2

Promedio obtenido por cada equipo en torno a la frecuencia de participación en cada foro de discusión en el curso IFP

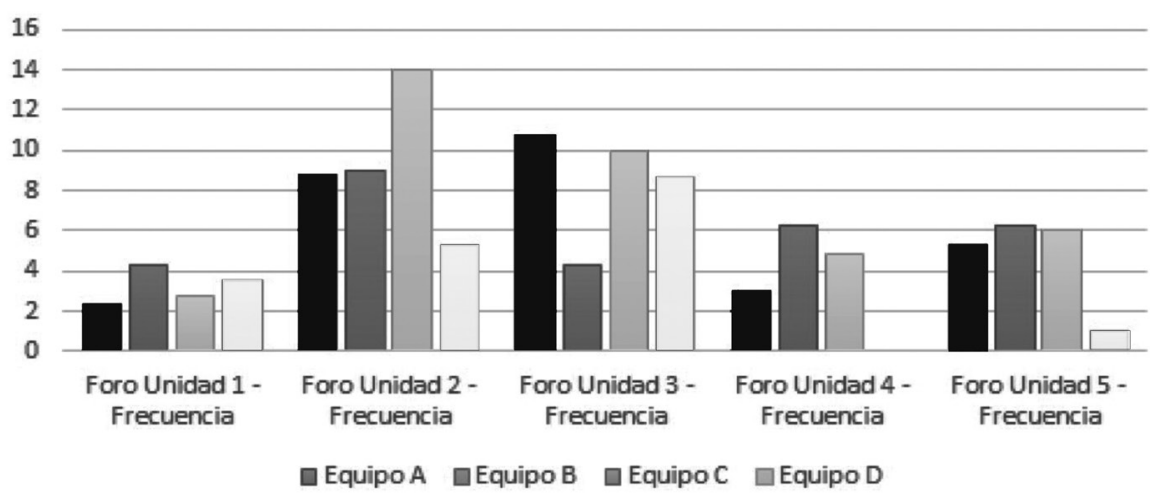

En la tabla 11 se aprecia que en el equipo D en las dos últimas sesiones prácticamente desapareció la actividad; el equipo B fue el que obtuvo una actividad más estable y el equipo $C$ tiene niveles de desempeño muy altos pero no estable.

\section{Calificación en el ensayo de equipo (nivel equipo-Curso STP)}

Se calculó la calificación del ensayo promediando el porcentaje de todas las calificaciones que el equipo recibió en la dinámica de co-evaluación, encon- 
trando que en el curso stP el equipo A y C tienen un patrón discontinuo en torno a las calificaciones de los ensayos. En el caso del equipo A, comienza con calificación alta (92), en el segundo ensayo baja (75), en el tercero aumenta notablemente (93), para volver a bajar en el último (78). En el caso del equipo D, la primera y la última calificaciones son las más altas (89 y 88 respectivamente), mientras que en el equipo $C$, sus primeras 2 calificaciones son altas, y las siguientes 2 son bajas (ver tabla 12 y gráfica 3 ).

Tabla 12

Muestra el promedio obtenido por cada equipo en la calificación del ensayo en el curso STP

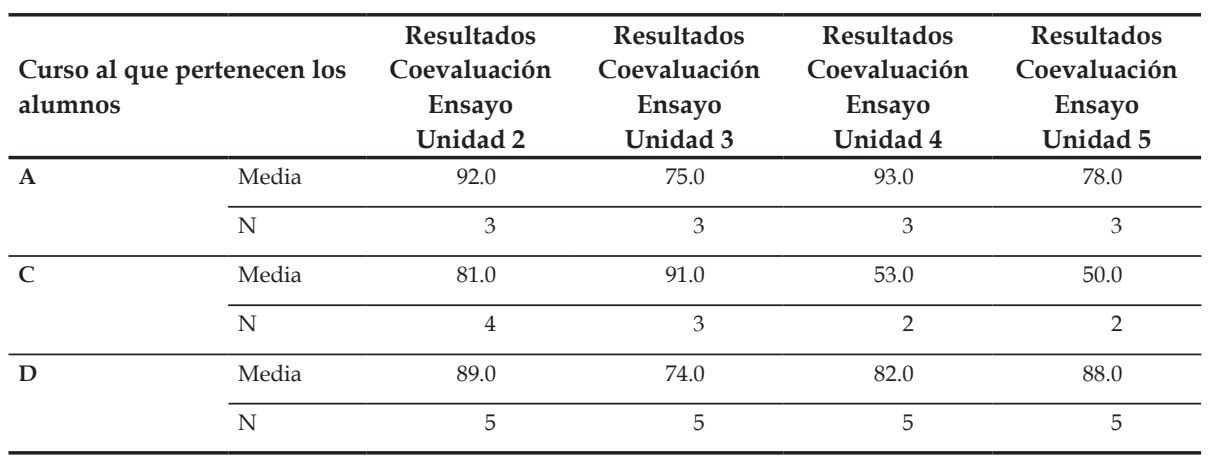

Gráfica 3

Promedio obtenido por cada equipo en la calificación del ensayo en el curso STP

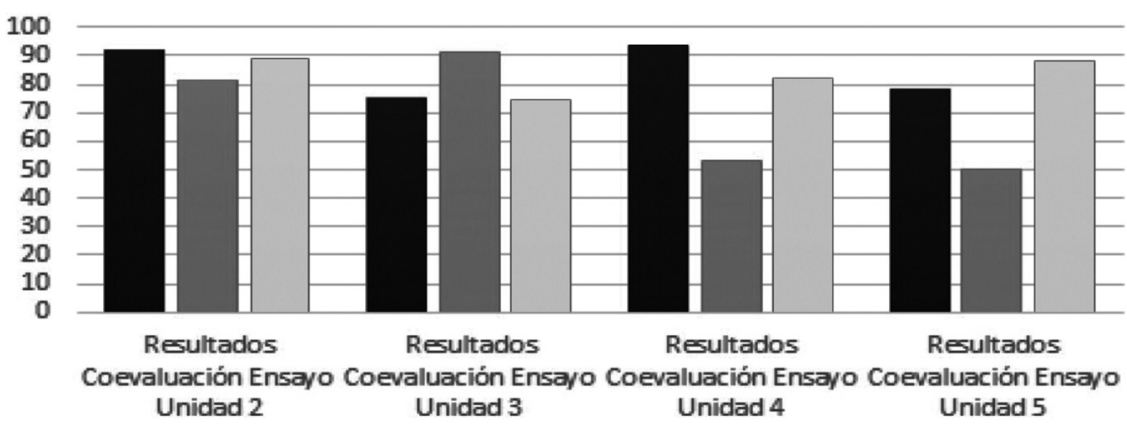

Un punto relevante de señalar es que el equipo C muestra una caída en las calificaciones de sus ensayos en las últimas unidades, lo que es consistente con lo observado en la actividad de los foros donde también en las últimas actividades mostró baja participación. Esta correspondencia ofrece un indicador de congruencia en los datos, indicando que cuando baja la actividad en los foros, parece bajar la calidad de los ensayos. De igual manera se aprecia 
que el equipo D obtiene buenas calificaciones en sus ensayos y también indicadores altos de participación en foro.

\section{Calificación en el ensayo de equipo (nivel equipo-Curso IFP)}

En el curso IFP se encontró que el Ensayo de la Unidad 2 y el de la Unidad 3 son los que presentan las calificaciones más altas en la mayoría de los equipos (excepto en el B). Encontramos también que los equipos con el desempeño más alto en sus ensayos fueron A y C (ver tabla 13 y gráfica 4).

Tabla 13

Muestra el promedio obtenido por cada equipo en la calificación del ensayo en el curso IFP

\begin{tabular}{lccccc}
\hline $\begin{array}{l}\text { Curso al que pertenecen los } \\
\text { alumnos }\end{array}$ & $\begin{array}{c}\text { Resultados } \\
\text { Coevaluación } \\
\text { Ensayo } \\
\text { Unidad 2 }\end{array}$ & $\begin{array}{c}\text { Resultados } \\
\text { Coevaluación } \\
\text { Ensayo } \\
\text { Unidad 3 }\end{array}$ & $\begin{array}{c}\text { Resultados } \\
\text { Coevaluación } \\
\text { Ensayo } \\
\text { Unidad 4 }\end{array}$ & $\begin{array}{c}\text { Resultados } \\
\text { Coevaluación } \\
\text { Ensayo } \\
\text { Unidad 5 }\end{array}$ \\
\hline A & Media & 86.0 & 100.0 & 56.0 & 67.0 \\
\cline { 2 - 6 } N & 3 & 3 & 3 & 3 \\
\hline Media & 79.0 & 33.0 & 56.0 & 53.0 \\
\hline N & Media & 88.0 & 75.0 & 94.0 & 42.0 \\
\cline { 2 - 6 } & $\mathrm{N}$ & 3 & 3 & 3 & 3 \\
\hline
\end{tabular}

Gráfica 4

Promedio obtenido por cada equipo en la calificación del ensayo en el curso IFP

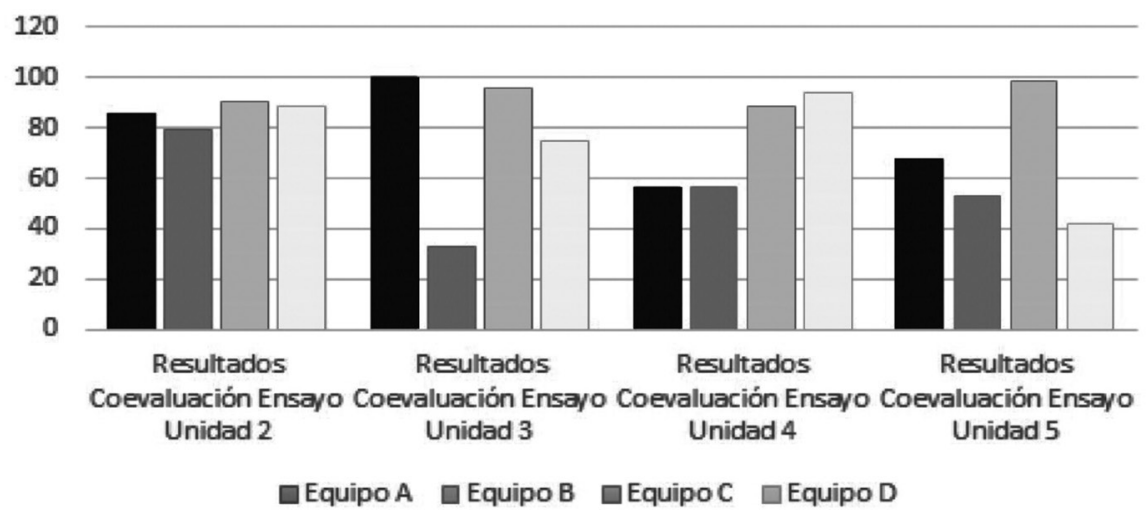

En el caso de los equipos del curso IFP no se observa una consistencia entre actividad en foros y la evaluación en los ensayos, pero hay que recordar que los contenidos curriculares de este curso son más voluminosos, heterogéneos 
y complejos, lo que de alguna manera puede dar elementos para explicar la inconsistencia.

\section{Tiempo en plataforma (nivel equipo-Curso STP)}

Se calculó el tiempo que los alumnos dedicaron al trabajo en el aula virtual, tomando como criterio si hubo menos de 15 minutos entre acciones (clics), encontrando que en el curso STP el equipo A fue el que más tiempo dedicó al aula virtual, seguido por el D y el C (ver tabla 14 y gráfica 5).

Tabla 14

Muestra el promedio obtenido por cada equipo en torno al tiempo de dedicación en el aula virtual en el curso STP

\begin{tabular}{llc}
\hline \multirow{2}{*}{$\begin{array}{l}\text { Equipo al que pertenecen } \\
\text { los alumnos }\end{array}$} & $\begin{array}{c}\text { Tiempo en } \\
\text { Plataforma } \\
\text { (Dedicación } \\
\text { en horas) }\end{array}$ \\
\hline A & Media & 28.8 \\
\cline { 2 - 3 } & $\mathrm{N}$ & 4 \\
\hline C & Media & 13.8 \\
\cline { 2 - 3 } & N & 3 \\
\hline D & Media & 25.7 \\
\cline { 2 - 3 } & $\mathrm{N}$ & 5 \\
\hline
\end{tabular}

Gráfica 5

Promedio obtenido por cada equipo en torno al tiempo de dedicación en el aula virtual en el curso STP Tiempo en Plataforma (Dedicación en horas)

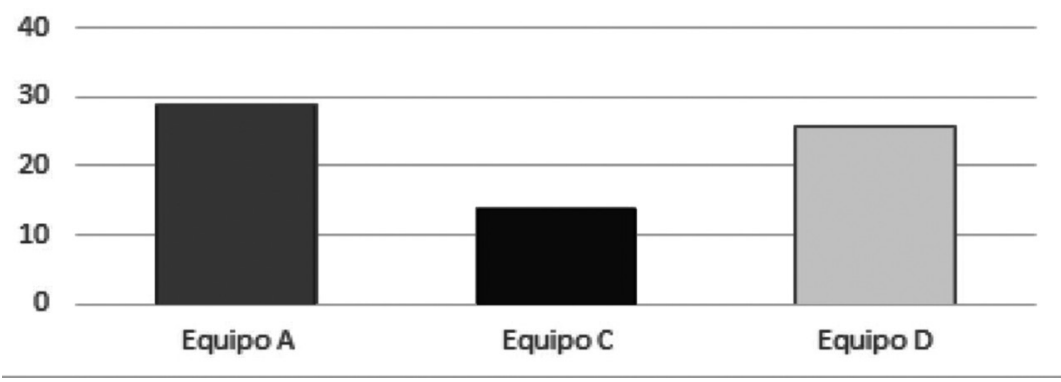

\section{Tiempo en plataforma (nivel equipo-Curso IFP)}

En esta dimensión se encontró que los equipos $\mathrm{A}$ y $\mathrm{C}$ fueron los que más tiempo dedicaron al trabajo en el aula virtual, lo cual es consistente con el desempeño observado en el proceso de la colaboración, la participación y la calificación en el ensayo (ver tabla 15 y gráfica 6). 
Tabla 15

Muestra el promedio obtenido por cada equipo en torno al tiempo de dedicación en el aula virtual en el curso IFP

\begin{tabular}{llr}
\hline \multirow{2}{*}{$\begin{array}{l}\text { Equipo al que pertenecen } \\
\text { los alumnos }\end{array}$} & $\begin{array}{c}\text { Tiempo en } \\
\text { Plataforma } \\
\text { (Dedicación } \\
\text { en horas) }\end{array}$ \\
\hline A & Media & 26.5 \\
\cline { 2 - 3 } & $\mathrm{N}$ & 4 \\
\hline B & Media & 20.9 \\
\cline { 2 - 3 } & $\mathrm{N}$ & 3 \\
\hline C & Media & 26.6 \\
\cline { 2 - 3 } & $\mathrm{N}$ & 4 \\
\hline D & Media & 18.8 \\
\cline { 2 - 3 } & $\mathrm{N}$ & 4 \\
\hline
\end{tabular}

\section{Gráfica 6}

Promedio obtenido por cada equipo en torno al tiempo de dedicación en el aula virtual en el curso IFP Tiempo en Plataforma (Dedicación en horas)

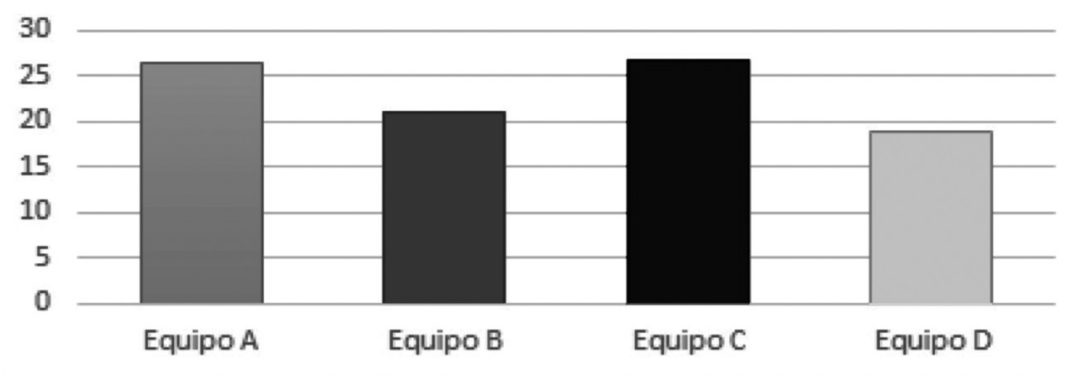

En términos generales, en el grupo IFP se observa también una consistencia entre número de participaciones en foro, la calificación obtenida en el ensayo y el tiempo de trabajo en plataforma. Aunque ciertamente no siempre se preserva la consistencia, en el caso del equipo D en el cuarto ensayo los registros en la base de datos indican que hubo 0 participación en foro en el caso del ensayo de la cuarta unidad, y sólo 1 en el caso de la quinta unidad. Estos datos probablemente no generan consistencia porque los alumnos pudieron haber trabajado fuera de plataforma y por lo mismo no se registraron los tiempos dedicados a la elaboración de este ensayo. Este tipo de problemas parecen ser naturales en una plataforma de educación a distancia, porque el trabajo no necesariamente tiene que estar restringido a la elaboración de productos 
en la plataforma, lo que sin duda agrega una fuente de vacío de información que muestra una debilidad del recurso tecnológico utilizado.

\section{Nivel Individuo}

Análisis de correlación (nivel individuo - ambos cursos)

Se realizó un análisis de correlación entre las tres dimensiones del proceso de colaboración: Organización del Trabajo (от), Contribución en la Elaboración del Ensayo (EE), Construcción de Conocimiento (CC). Además se correlacionó con la frecuencia de participación, la calificación de los mapas conceptuales, la de los ensayos, el tiempo que se trabajó en el aula virtual, así como el promedio final del curso.

Las tres dimensiones del proceso de colaboración estuvieron fuertemente relacionadas entre sí, dadas las correlaciones estadísticamente significativas que presentaron entre ellas. La dimensión de Organización del Trabajo (От) tuvo correlaciones altas y estadísticamente significativas con la calificación de mapas conceptuales y la de promedio de los ensayos de equipo, lo cual indica que quien tendió a organizar acuerdos relacionados con la coordinación de las actividades, presentó mejores productos de aprendizaje.

La frecuencia de participación en las discusiones guardó una estrecha relación con la calificación de mapas conceptuales, lo que indica que quien intercambió mensajes con más constancia, en general entregó mejores productos de aprendizaje individuales.

Las tres correlaciones que más destacan son las observadas entre la calificación de mapas conceptuales, ensayos y tiempo en plataforma, lo cual indica que una mayor dedicación a las actividades del aula derivó en mejores productos individuales de aprendizaje y de equipo. Este resultado apoya una tesis muy señalada, que consiste en que los estudiantes que tienen mejor disciplina de trabajo, también identificada como auto-regulación, son quienes obtienen los mejores resultados (ver tabla 16).

Lo más relevante a observar en la tabla de correlaciones es observar que en la calificación promedio del curso todos los altos valores son altos y estadísticamente significativos ( ${ }^{*}=p<0.01$ ), lo cual implica que hay una consistencia de los diversos factores observados con la calificación final. En particular son muy altos con relación a la calificación de mapas conceptuales, de los ensayos y del tiempo en plataforma, lo cual también es muy congruente, en tanto esto indica que quienes más trabajaron (tiempo en plataforma) hicieron mejores mapas conceptuales y ensayos. 


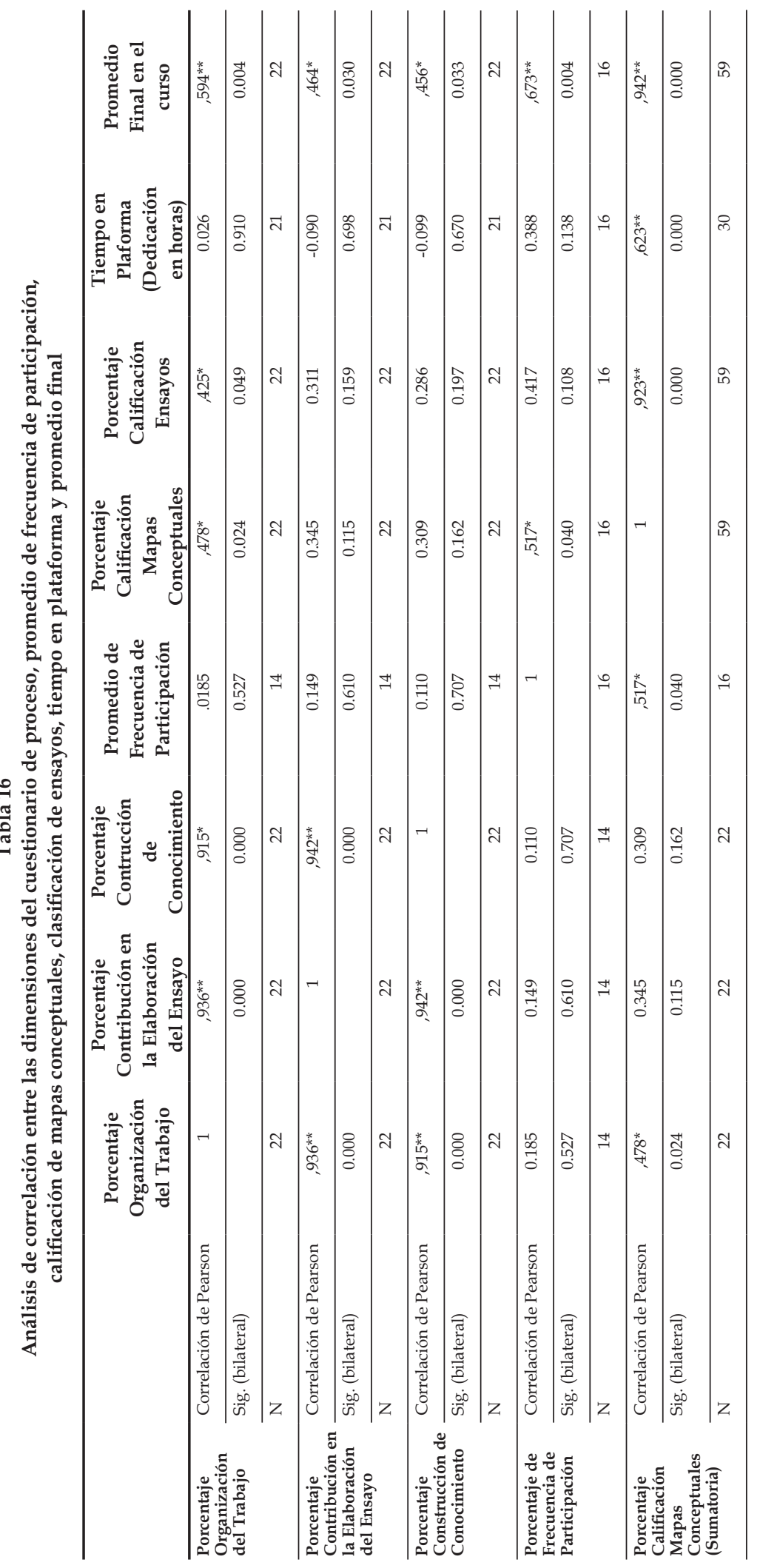




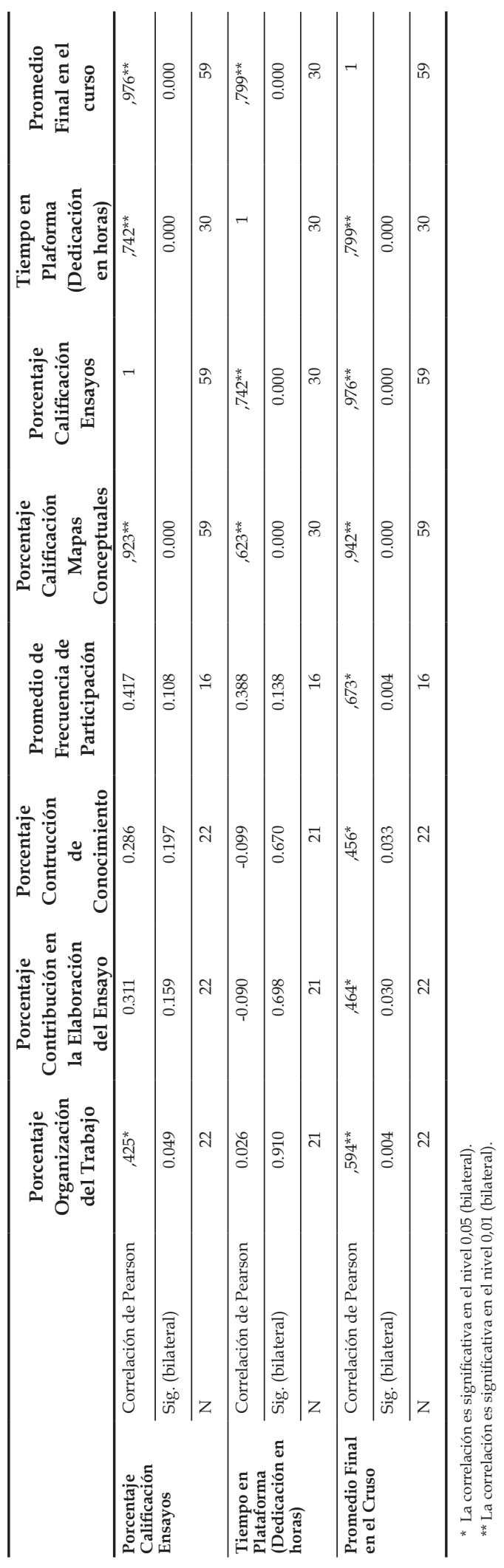


Las altas correlaciones observadas indican la coherencia psicométrica y están a favor del diseño educativo implementado.

\section{Discusión y conclusiones}

De acuerdo con los resultados obtenidos en esta experiencia de investigación, se mostró útil emplear distintos niveles de análisis (curso-equipo-alumno) para tener una mejor comprensión respecto al desempeño del diseño educativo empleado, partiendo de diversas métricas relacionadas con procesos y resultados (productos) de aprendizaje tanto a nivel individual como de equipo.

De acuerdo a las hipótesis planteadas, en la primera de ellas se formuló que: Existen tendencias de desempeño en el cuestionario del proceso de la colaboración, de acuerdo al grupo, el equipo y el alumno, encontrando que efectivamente las hay.

De acuerdo a la segunda hipótesis: Existen tendencias de desempeño en la participación en los foros para la construcción del ensayo de acuerdo al curso, equipo y alumno. Esta hipótesis resulta parcialmente cierta, en tanto se observa un efecto de agotamiento en los cursos, sin embargo, es interesante que este agotamiento, cuando se analiza a nivel de equipos y de alumnos, no se da en todos. Por lo mismo, es importante observar en el desglose de los datos que hay composiciones distintas de acuerdo al nivel de análisis (curso, equipo, alumno).

En la tercera hipótesis se sostiene que: Existen tendencias de desempeño en torno a la calificación obtenida en los ensayos de acuerdo al curso, el equipo y el alumno. Efectivamente se observa que el desempeño no presenta grandes tendencias en los cursos, pero sí en los equipos y alumnos, ya que hay equipos y alumnos de bajo, medio y alto desempeños dada la diversidad de calificaciones obtenidas. Este resultado invita a que haya una mejor planeación de la distribución de los alumnos en los equipos de trabajo, de manera que sean más homogéneos, integrando alumnos tanto de bajo, mediano y alto desempeños en cada uno de los equipos, tomando en cuenta su historia (promedios escolares y ritmos/reprobación).

En relación a la cuarta hipótesis, se plantea que: Existen tendencias de desempeño en torno al tiempo que los alumnos invierten en la plataforma de acuerdo al grupo, el equipo y el alumno. Nuevamente es interesante observar que al nivel de los cursos no se observan grandes diferencias, en contraste, al analizar los equipos de trabajo sí se aprecia que unos tuvieron más tiempo de trabajo que otros. Los procesos educativos son sistémicos, se interrelacionan múltiples factores en los que unos inhiben o promueven diversos efectos, de aquí que resulte entendible que cuando en un equipo no hay una alta tasa de des- 
empeño por parte de sus integrantes, se crea un ambiente que desanima al trabajo colectivo.

En lo que respecta a la quinta hipótesis, se plantea que: Existe una relación entre el desempeño en la elaboración de productos de aprendizaje de equipo y alumno, con la evaluación del proceso de la colaboración. De lo anterior se observó que existe una dimensión del cuestionario de evaluación del proceso de colaboración (Organización del Trabajo de Equipo-OT) que está estrechamente relacionada con el desempeño y los productos de aprendizaje de los equipos e individuos, por lo que podemos decir que esta hipótesis se cumple parcialmente, en tanto sólo se relaciona con esta dimensión del cuestionario.

Para finalizar, en la última hipótesis se plantea que: Existe una relación entre el desempeño en la elaboración de productos de aprendizaje de equipo y alumno, con el tiempo de actividad en el aula virtual. En este aspecto pudimos observar que efectivamente el tiempo que los alumnos invierten en las actividades de la plataforma, se relaciona estrechamente con la calificación de sus productos de aprendizaje individuales y de equipo. Lo anterior es relevante, pues el diseño educativo utilizado en esta experiencia supone una participación intensiva en actividades que se llevan a cabo exclusivamente en el aula virtual, usándola como vehículo para elaborar productos de aprendizaje (sobre todo los de equipo) y también para llevar a cabo intercambios comunicativos, relacionados con el proceso de elaboración de estos productos.

Es importante mencionar que se descartaron necesariamente de la mayor parte de los análisis, aquellos alumnos que por haber desertado tempranamente, o no concluir el curso, no se tenían datos en la mayor parte de las actividades. En el caso del módulo Sistemas Teóricos de la Psicología (STP) dejaron de participar 11 alumnos y en el de Introducción a la Filosofía de la Psicología (IFP) 25, donde se observa, como ya se ha expuesto, que el abandono suele ser frecuente en los sistemas de educación a distancia.

En cuanto a las implicaciones en el diseño educativo, es importante mencionar que un aspecto que debe cuidarse en los entornos virtuales de aprendizaje, es no saturar de actividades a los estudiantes, más considerando que muchos de ellos trabajan y son padres de familia. Es mejor obtener esfuerzos constantes y persistentes de participación en las actividades, que forzar al cumplimiento de múltiples tareas que resulten en un agotamiento que pudiese llevar a la deserción. Se requiere promover en los programas de educación a distancia, un modelo educativo que se acople al perfil socio-demográfico de los estudiantes, con diseños de experiencias de aprendizaje basadas en las características de éstos, de modo que puedan aprovechar mejor sus potencialidades. 
Por último, es posible plantear diseños educativos que promuevan la actividad epistémica de construcción de conocimiento, bajo la argumentación y la contra-argumentación, como se puede dar en los diseños de aprendizaje por colaboración basados en la co-enseñanza y co-evaluación, de manera tal que se transforme el proceso educativo, es decir, se trata de transformar la educación.

\section{Referencias}

Barab, S. (2014). Design-Based Research. En R. K. Sawyer (Ed.), The Cambridge Handbook of the Learning Sciences (2.a ed., pp. 151-170). Cambridge: Cambridge University Press. Recuperado a partir de http:/ / ebooks.cambridge.org/ref/ id/CBO9781139519526A017

Cohen, E. G. (1994). Restructuring the Classroom: Conditions for Productive Small Groups. Review of Educational Research, 64 (1), 1-35. https://doi. org/10.3102/00346543064001001

Cole, M., \& Engeström, Y. (1993). A cultural-historical approach to distributed cognition. Distributed cognitions: Psychological and educational considerations, 1-46.

Coll, C., Bustos, A., \& Engel, A. (2015). La información sobe el ejercicio de la influencia educativa como medio para favorecer la participación y el aprendizaje en un foro en línea. Infancia y Aprendizaje: Journal for the Study of Education and Development, 38 (2), 368-401.

Coll, C., Mauri, T., \& Onrubia, J. (2008). La utilización de las tecnologías de la información y la comunicación en la educación: Del diseño tecno-pedagógico a las prácticas de uso. En C. Coll \& C. Monereo (Eds.), Psicología de la Educación Virtual (pp. 74-103). Ediciones Morata.

Coll, C., Onrubia, J., \& Majós, T. M. (2008). Ayudar a aprender en contextos educativos: el ejercicio de la influencia educativa y el análisis de la enseñanza. Revista de educación, (346), 33-70.

Collins, A. (2006). Cognitive apprenticeship. En K. Sawyer (Ed.), The Cambridge handbook of the learning sciences (pp. 47-60). Cambridge University Press.

Collins, A., Brown, J. S., \& Holum, A. (1991). Cognitive apprenticeship: making thinking visible. American Educator, 6, 38-46.

Colomina, R., Onrubia, J., \& Rochera, M. J. (2007). Interactividad, mecanismos de influencia educativa y construcción del conocimiento en el aula. En C. Coll, J. Palacios, \& Á. Marchesi (Eds.), Desarrollo psicológico y educación: 2. Psicología de la educación escolar (pp. 437-456). Alianza Editorial, S. A.

Dennen, V., \& Hoadley, C. (2013). Designing Collaborative Learning through Computer Support. En C. E. Hmelo-Silver (Ed.), The international handbook of collaborative learning (pp. 389-402).

Design-Based Research: An Emerging Paradigm for Educational Inquiry. (2003). Educational Researcher,32(1),5-8.https:/ / doi.org/10.3102/0013189X032001005 
Dillenbourg, P. (1999). What do you mean by â $\square \square$ collaborative learning'? Collaborative learning Cognitive and computational approaches, 1 (6), 1-15. https:/ / doi. org/10.1.1.167.4896

Dillenbourg, P. (2002). Over-scripting CSCL : The risks of blending collaborative learning with instructional design. Three worlds of CSCL Can we support CSCL, 117 (6), 61-91.

Dillenbourg, P. (2005). Designing Biases That Augment Socio-Cognitive Interactions. En R. Bromme \& H. Spada (Eds.), Barriers and Biases in Computer-Mediated Knowledge Communication (Vol. 5, pp. 243-264). Springer US. Recuperado a partir de http://www.springerlink.com/content/u077239317657586/abstract/

Dillenbourg, P., Järvelä, S., \& Fischer, F. (2009). The Evolution of Research on Computer-Supported Collaborative Learning. En N. Balacheff, S. Ludvigsen, T. Jong, A. Lazonder, \& S. Barnes (Eds.), Technology-Enhanced Learning (pp. 3-19). Springer Netherlands. Recuperado a partir de http://www.springerlink.com.pbidi.unam.mx:8080/content/x4687xr712341w22/abstract/

Dillenbourg, P., \& Jermann, P. (2007). Designing Integrative Scripts. En F. Fischer, I. Kollar, H. Mandl, \& J. M. Haake (Eds.), Scripting Computer-Supported Collaborative Learning (Vol. 6, pp. 275-301). Springer US. Recuperado a partir de http://www.springerlink.com.pbidi.unam.mx:8080/content/ whk6t97755313n3j/abstract/

Greeno, J. (2006). Learning in Activity. En R. K. Sawyer (Ed.), The Cambridge handbook of the learning sciences (pp. 79-96). Cambridge University Press.

Greeno, J. G. (1998). The Situativity of Knowing, Learning, and Research. American Psychologist, 53(1), 5-26.

Gunawardena, C. N., Lowe, C. A., \& Anderson, T. (1997). Analysis of a Global Online Debate and the Development of an Interaction Analysis Model for Examining Social Construction of Knowledge in Computer Conferencing. Journal of Educational Computing Research, 17 (4), 397-431.

Jermann, P., Soller, A., \& Muehlenbrock. (2005). From mirroring to guiding: a review of the state of the art technology for supporting collaborative learning. Recuperado a partir de http://citeseerx.ist.psu.edu/viewdoc/ summary?doi=10.1.1.26.2186

Kirschner, P. A. (2002). Can we support cscL? Educational, social and technological affordances for learning. En Three Words of cscl Can we Support CSCL (p. 132).

Kirschner, P., Martens, R., \& Strijbos, J.-W. (2004). cscL in higher education: a framework for designing multiple collaborative environments. Kluwer Academic Publishers. Recuperado a partir de http://portal.acm.org/citation. cfm?id=1011199.1011202

Kobbe, L., Weinberger, A., Dillenbourg, P., Harrer, A., Hämäläinen, R., Häkkinen, P., \& Fischer, F. (2007). Specifying computer-supported collaboration scripts. International Journal of Computer-Supported Collaborative Learning, 2 (2-3), 211224. https://doi.org/10.1007/s11412-007-9014-4

Kollar, I., Fischer, F., \& Hesse, F. (2006). Collaboration Scripts-A Conceptual Analysis. Educational Psychology Review, 18 (2), 159-185. https://doi. org/10.1007/s10648-006-9007-2 
Laat, M. (2002). Network and Content Analysis in an Online Community Discourse. En In: G. Stahl (Ed.), Proceedings of Computer Support for Collaborative Learning (CSCL) 2002 Conference (pp. 625-626). Erlbaum.

Määttä, E., Järvenoja, H., \& Järvelä, S. (2012). Triggers of Students' Efficacious Interaction in Collaborative Learning Situations. Small Group Research, 43 (4), 497-522. https:/ / doi.org/10.1177/1046496412437208

Meyer, K. A. (2014). Student Engagement in Online Learning: What Works and Why. ASHE Higher Education Report, 40 (6), 1-114. https://doi.org/10.1002/ aehe. 20018

Novak, J. D., \& Cañas, A. J. (2006). The theory underlying concept maps and how to construct them. Florida Institute for Human and Machine Cognition, 1.

O’Donnell, A. M., \& King, A. (1999). Cognitive Perspectives on Peer Learning. Taylor \& Francis.

Palincsar, A. S., \& Brown, A. L. (1986). Interactive Teaching to Promote Independent Learning from Text. The Reading Teacher, 39 (8), 771-777.

Pea, R. (1997). Practices of distributed intelligence and designs for education. En Distributed Cognitions: Psychological and Educational Considerations (pp. 47-87). Cambridge University Press. Recuperado a partir de http://www.amazon. fr/exec/obidos/ASIN/0521574234/citeulike04-21

Ploetzner, R., Dillenbourg, P., Preier, M., \& Traum, D. (1999). Learning by Explaining to Oneself and to Others. En P. Dillenbourg (Ed.), Collaborative learning: cognitive and computational approaches (pp. 103-121). Oxford: Elsevier.

Reigeluth, C. M. (2000). Diseño de la Instrucción: Teorías y Modelos: Un Nuevo Paradigma de la Teoría de la Instrucción. Alfaguara, Ediciones, S.A.-Grupo Santillana.

Roschelle, J., \& Teasley, S. D. (1995). The construction of shared knowledge in collaborative problem solving. Knowledge Creation Diffusion Utilization, 128 (3), 69-97. https:// doi.org/10.1145/130893.952914

Salomon, G. (2001). Cogniciones Distribuidas: Consideraciones Psicologicas y Educativas (Colección Agenda Educativa). Amorrortu Editores.

Sawyer, K. (2006). The New Science of Learning. En K. Sawyer (Ed.), Cambridge Handbook of the Learning Sciences (pp. 97-119). Cambridge, MA: Harvard University Press.

Sawyer, K. (2008). Optimising Learning Implications of Learning Sciences Research (pp. 1-14). Presentado en International Conference «Learning in the 21st Century: Research, Innovation and Policy», Centre for Educational Research and Innovation: Organization for Economic Co-operation and Development. Recuperado a partir de http:/ / www.ingentaconnect.com/content/oe cd/16080165/2008/00002008/00000021/9608091ec004

Scardamalia, M. (2002). Collective cognitive responsibility for the advancement of knowledge. En B. Smith (Ed.), Liberal education in a knowledge society (pp. 67-98). Chicago: Open Court.

Scardamalia, M., \& Bereiter, C. (2006). Knowledge Building: Theory, Pedagogy, and Technology. En K. Sawyer (Ed.), Cambridge Handbook of the Learning Sciences (pp. 97-119). Cambridge University Press. 
Sharan, S. (1980). Cooperative Learning in Small Groups: Recent Methods and Effects on Achievement, Attitudes, and Ethnic Relations. Review of Educational Research, 50 (2), 241-271. https:/ / doi.org/10.3102/00346543050002241

Stahl, G., Koschmann, T., \& Suthers, D. (2006). Computer-supported collaborative learning: An Historical Perspective. En Cambridge handbook of the learning sciences (pp. 409-426). Cambridge University Press. Recuperado a partir de http:/ / citeseerx.ist.psu.edu/viewdoc/summary?doi=10.1.1.63.1418

Strijbos, J. W., Martens, R. L., \& Jochems, W. M. G. (2004). Designing for interaction: Six steps to designing computer-supported group-based learning. Computers E Education, 42 (4), 403-424. https://doi.org/10.1016/j.compedu.2003.10.004

Tudge, J. R. H. (1992). Processes and Consequences of Peer Collaboration: A Vygotskian Analysis. Child Development, 63 (6), 1364-1379. https://doi. org/10.1111/j.1467-8624.1992.tb01701.x

Toulmin, S. E. (2003). The uses of argument. Cambridge university press.

Webb, N. M. (1989). Peer interaction and learning in small groups. International Journal of Educational Research, 13 (1), 21-39. https:// doi.org/10.1016/08830355(89)90014-1

Weinberger, A., Stegmann, K., \& Fischer, F. (2007). Knowledge convergence in collaborative learning: Concepts and assessment. Learning and Instruction, 17 (4), 416-426. https:// doi.org/10.1016/j.learninstruc.2007.03.007 
